\title{
Nonzero-Sum Stochastic Differential Game between Controller and Stopper for Jump Diffusions
}

\author{
Yan Wang, ${ }^{1,2}$ Aimin Song, ${ }^{2}$ Cheng-De Zheng, ${ }^{2}$ and Enmin Feng ${ }^{1}$ \\ ${ }^{1}$ School of Mathematical Sciences, Dalian University of Technology, Dalian 116023, China \\ ${ }^{2}$ School of Science, Dalian Jiaotong University, Dalian 116028, China \\ Correspondence should be addressed to Yan Wang; wymath@163.com
}

Received 5 February 2013; Accepted 7 May 2013

Academic Editor: Ryan Loxton

Copyright (c) 2013 Yan Wang et al. This is an open access article distributed under the Creative Commons Attribution License, which permits unrestricted use, distribution, and reproduction in any medium, provided the original work is properly cited.

\begin{abstract}
We consider a nonzero-sum stochastic differential game which involves two players, a controller and a stopper. The controller chooses a control process, and the stopper selects the stopping rule which halts the game. This game is studied in a jump diffusions setting within Markov control limit. By a dynamic programming approach, we give a verification theorem in terms of variational inequality-Hamilton-Jacobi-Bellman (VIHJB) equations for the solutions of the game. Furthermore, we apply the verification theorem to characterize Nash equilibrium of the game in a specific example.
\end{abstract}

\section{Introduction}

In this paper we study a nonzero-sum stochastic differential game with two players: a controller and a stopper. The state $X(\cdot)$ in this game evolves according to a stochastic differential equation driven by jump diffusions. The controller affects the control process $u(\cdot)$ in the drift and volatility of $X(\cdot)$ at time $t$, and the stopper decides the duration of the game, in the form of a stopping rule $\tau$ for the process $X(\cdot)$. The objectives of the two players are to maximize their own expected payoff.

In order to illustrate the motivation and the background of application for this game, we show a model in finance.

Example 1. Let $\left(\Omega, \mathscr{F},\left\{\mathscr{F}_{t}\right\}_{t \geq 0}, P\right)$ be a filtered probability space, let $B(t)$ be a $k$-dimensional Brownian Motion, and let $\widetilde{N}(d t, d z)=\left(\widetilde{N}_{1}(d t, d z), \ldots, \widetilde{N}_{k}(d t, d z)\right)$ be $k$-independent compensated Poisson random measures independent of $B(t)$, $t \in[0, \infty)$. For $i=1, \ldots, k, \widetilde{N}_{i}(d t, d z)=N_{i}(d t, d z)-v_{i}(d z) d t$, where $v_{i}$ is the Lévy measure of a Lévy process $\eta_{i}(t)$ with jump measure $N_{i}$ such that $E\left[\eta_{i}^{2}(t)\right]<\infty$ for all $t .\left\{\mathscr{F}_{t}\right\}_{t \geq 0}$ is the filtration generated by $B(t)$ and $\widetilde{N}(d t, d z)$ (as usual augmented with all the $P$-null sets). We refer to $[1,2]$ for more information about Lévy processes.
We firstly define a financial market model as follows. Suppose that there are two investment possibilities:

(1) a risk-free asset (e.g., a bond), with unit price $S_{0}(t)$ at time $t$ given by

$$
d S_{0}(t)=r(t) S_{0}(t) d t, \quad S_{0}(0)=1,
$$

(2) a risky asset (e.g., a stock), with unit price $S_{1}(t)$ at time $t$ given by

$d S_{1}(t)$

$$
\begin{array}{r}
=S_{1}(t-)\left[b(t) d t+\sigma(t) d B(t)+\int_{\mathbb{R}} \gamma(t, z) \widetilde{N}(d t, d z)\right], \\
S_{1}(0)>0,
\end{array}
$$

where $r(t)$ is $\mathscr{F}_{t}$-adapted with $\int_{0}^{T}|r(t)| d t<\infty$ a.s., $T>0$ is a fixed given constant, $b, \sigma, \gamma$ is $\mathscr{F}_{t}$-predictable processes satisfying $\gamma(t, z)>-1$ for a.a. $t, z$, a.s. and

$$
\int_{0}^{T}\left\{|b(t)|+\sigma^{2}(t)+\int_{\mathbb{R}} \gamma^{2}(t, z) \nu(d z)\right\} d t<\infty, \quad \forall T<\infty .
$$


Assume that an investor hires a portfolio manager to manage his wealth $X(t)$ from investments. The manager (controller) can choose a portfolio $u(t)$, which represents the proportion of the total wealth $X(t)$ invested in the stock at time $t$. And the investor (stopper) can halt the wealth process $X(t)$ by selecting a stop-rule $\tau: C[0, T] \rightarrow[0, T]$. Then the dynamics of the corresponding wealth process $X(t)=X^{u}(t)$ is

$$
\begin{array}{r}
d X^{u}(t)=X^{u}(t-)\{[(1-u(t)) r(t)+u(t) b(t)] d t \\
+u(t) \sigma(t) d B(t) \\
\left.+u(t-) \int_{\mathbb{R}} \gamma(t, z) \widetilde{N}(d t, d z)\right\}, \\
X^{u}(0)=x>0
\end{array}
$$

(see, e.g., [3-5]). We require that $u(t-) \gamma(t, z)>-1$ for a.a. $t, z$, a.s. and that

$$
\begin{gathered}
\int_{0}^{T}\left\{|(1-u(t)) r(t)|+|u(t) b(t)|+u^{2}(t) \sigma^{2}(t)\right. \\
\left.+u^{2}(t) \int_{\mathbb{R}} \gamma^{2}(t, z) \nu(d z)\right\} d t<\infty \text { a.s. }
\end{gathered}
$$

At terminal time $\tau$, the stopper gives the controller a payoff $C(X(\tau))$, where $C: C[0, T] \rightarrow \mathbb{R}$ is a deterministic mapping. Therefore, the controller aims to maximize his utility of the following form:

$$
\mathscr{J}_{1}^{x}(u, \tau)=E^{x}\left[e^{-\delta \tau} U_{1}(C(X(\tau)))-\int_{0}^{\tau} e^{-\delta t} h\left(t, X(t), u_{t}\right) d t\right],
$$

where $\delta>0$ is the discounting rate, $h$ is a cost function, and $U_{1}$ is the controller's utility. We denote $E^{x}$ the expectation with respect to $P^{x}$ and $P^{x}$ the probability laws of $X(t)$ starting at $x$.

Meanwhile it is the stopper's objective to choose the stopping time $\tau$ such that his own utility

$$
\mathcal{F}_{2}^{x}(u, \tau)=E^{x}\left[e^{-\delta \tau} U_{2}\{X(\tau)-C(X(\tau))\}\right]
$$

is maximized, where $U_{2}$ is the stopper's utility.

As this game is typically a nonzero sum, we seek a Nash equilibrium, namely, a pair $\left(u^{*}, \tau^{*}\right)$ such that

$$
\begin{array}{ll}
\mathscr{J}_{1}^{x}\left(u^{*}, \tau^{*}\right) \geq \mathscr{J}_{1}^{x}\left(u, \tau^{*}\right), & \forall u, \\
\mathscr{J}_{2}^{x}\left(u^{*}, \tau^{*}\right) \geq \mathscr{J}_{2}^{x}\left(u^{*}, \tau\right), \quad \forall \tau .
\end{array}
$$

This means that the choice $u^{*}$ is optimal for the controller when the stopper uses $\tau^{*}$ and vice verse.

The game (4) and (8) are a nonzero-sum stochastic differential game between a controller and a stopper. The existence of Nash equilibrium shows that, by an appropriate stopping rule design $\tau^{*}$, the stopper can induce the controller to choose the best portfolio he can. Similarly, by applying a suitable portfolio $u^{*}$, the controller can force the stopper to stop the employment relationship at a time of the controller's choosing.
There have been significant advances in the research of stochastic differential games of control and stopping. For example, in [6-9] the authors considered the zero-sum stochastic differential games of mixed type with both controls and stopping between two players. In these games, each of the players chooses an optimal strategy, which is composed by a control $u(\cdot)$ and a stopping $\tau$. Under appropriate conditions, they constructed a saddle pair of optimal strategies. For the nonsum case, the games of mixed type were discussed in $[6,10]$. The authors presented Nash equilibria, rather than saddle pairs, of strategies [10]. Moreover, the papers [11-17] considered a zero-sum stochastic differential game between controller and stopper, where one player (controller) chooses a control process $u(\cdot)$ and the other (stopper) chooses a stopping $\tau$. One player tries to maximize the reward and the other to minimize it. They presented a saddle pair for the game.

In this paper, we study a nonzero-sum stochastic differential game between a controller and a stopper. The controller and the stopper have different payoffs. The objectives of them are to maximize their own payoffs. This game is considered in a jump diffusion context under the Markov control condition. We prove a verification theorem in terms of VIHJB equations for the game to characterize Nash equilibrium.

Our setup and approach are related to [3, 17]. However, their games are different from ours. In [3], the authors studied the stochastic differential games between two controllers. The work in [17] was carried out for a zero-sum stochastic differential game between a controller and a stopper.

The paper is organized as follows: in the next section, we formulate the nonzero-sum stochastic differential game between a controller and a stopper and prove a general verification theorem. In Section 3, we apply the general results obtained in Section 2 to characterize the solutions of a special game. Finally, we conclude this paper in Section 4.

\section{A Verification Theorem for Nonzero-Sum Stochastic Differential Game between Controller and Stopper}

Suppose the state $Y(t)=Y^{u}(t)$ at time $t$ is given by the following stochastic differential equation:

$$
\begin{array}{r}
d Y(t)=\alpha\left(Y(t), u_{0}(t)\right) d t+\beta\left(Y(t), u_{0}(t)\right) d B(t) \\
+\int_{\mathbb{R}_{0}^{k}} \theta\left(Y(t-), u_{1}(t-, z), z\right) \widetilde{N}(d t, d z), \\
Y(0)=y \in \mathbb{R}^{k},
\end{array}
$$

where $\alpha: \mathbb{R}^{k} \times \mathfrak{U} \rightarrow \mathbb{R}^{k}, \beta: \mathbb{R}^{k} \times \mathfrak{U} \rightarrow \mathbb{R}^{k \times k}, \theta: \mathbb{R}^{k} \times$ $\mathfrak{U} \times \mathbb{R}^{k} \rightarrow \mathbb{R}^{k \times k}$ are given functions, and $\mathcal{U}$ denotes a given subset of $\mathbb{R}^{p}$.

We regard $u_{0}(t)=u_{0}(t, \omega)$ and $u_{1}(t, z)=u_{1}(t, z, \omega)$ as the control processes, assumed to be càdlàg, $\mathscr{F}_{t}$-adapted and with values in $u_{0}(t) \in \mathcal{U}, u_{1}(t, z) \in \mathcal{U}$ for a.a. $t, z, \omega$. And we put $u(t)=\left(u_{0}(t), u_{1}(t, z)\right)$. Then $Y(t)=Y^{(u)}(t)$ is a controlled jump diffusion (see [18] for more information about stochastic control of jump diffusion). 
Fix an open solvency set $\mathcal{S} \subset \mathbb{R}^{k}$. Let

$$
\tau_{\mathcal{S}}=\inf \{t>0 ; Y(t) \notin \mathcal{S}\}
$$

be the bankruptcy time. $\tau_{\mathcal{\delta}}$ is the first time at which the stochastic process $Y(t)$ exits the solvency set $\mathcal{S}$. Similar optimal control problems in which the terminal time is governed by a stopping criterion are considered in [19-21] in the deterministic case.

Let $f_{i}: \mathbb{R}^{k} \times K \rightarrow \mathbb{R}$ and $g_{i}: \mathbb{R}^{k} \rightarrow \mathbb{R}$ be given functions, for $i=1,2$. Let $\mathscr{A}$ be a family of admissible controls, contained in the set of $u(\cdot)$ such that (9) has a unique strong solution and

$$
E^{y}\left[\int_{0}^{\tau_{\delta}}\left|f_{i}\left(Y_{t}, u_{t}\right)\right| d t\right]<\infty, \quad i=1,2
$$

for all $y \in \mathcal{S}$, where $E^{y}$ denotes expectation given that $Y(0)=$ $y \in \mathbb{R}^{k}$. Denote by $\Gamma$ the set of all stopping times $\tau \leq \tau_{\mathcal{\delta}}$. Moreover, we assume that

the family $\left\{g_{i}^{-}\left(Y_{\tau}\right) ; \tau \in \Gamma\right\}$ is uniformly integrable,

$$
\text { for } i=1,2 \text {. }
$$

Then for $u \in \mathscr{A}$ and $\tau \in \Gamma$ we define the performance functionals as follows:

$$
\begin{array}{r}
\mathscr{J}_{i}^{y}(u, \tau)=E^{y}\left[\int_{0}^{\tau} f_{i}(Y(t), u(t)) d t+g_{i}(Y(\tau))\right], \\
i=1,2 .
\end{array}
$$

We interpret $g_{i}(Y(\tau))$ as 0 if $\tau=\infty$. We may regard $\mathscr{J}_{1}^{y}(u, \tau)$ as the payoff to the controller who controls $u$ and $\mathscr{J}_{2}^{y}(u, \tau)$ as the payoff to the stopper who decides $\tau$.

Definition 2 (nash equilibrium). A pair $\left(u^{*}, \tau^{*}\right) \in \mathscr{A} \times \Gamma$ is called a Nash equilibrium for the stochastic differential game (9) and (13), if the following holds:

$$
\begin{aligned}
& \mathscr{J}_{1}^{y}\left(u^{*}, \tau^{*}\right) \geq \mathscr{J}_{1}^{y}\left(u, \tau^{*}\right), \quad \forall u \in \mathcal{U}, y \in \mathcal{S}, \\
& \mathscr{J}_{2}^{y}\left(u^{*}, \tau^{*}\right) \geq \mathscr{J}_{2}^{y}\left(u^{*}, \tau\right), \quad \forall \tau \in \Gamma, y \in \mathcal{S} .
\end{aligned}
$$

Condition (14) states that if the stopper chooses $\tau^{*}$, it is optimal for the controller to use the control $u^{*}$. Similarly, condition (15) states that if the controller uses $u^{*}$, it is optimal for the stopper to decide $\tau^{*}$. Thus, $\left(u^{*}, \tau^{*}\right)$ is an equilibrium point in the sense that there is no reason for each individual player to deviate from it, as long as the other player does not.

We restrict ourselves to Markov controls; that is, we assume that $u_{0}(t)=\tilde{u}_{0}(Y(t)), u_{1}(t, z)=\tilde{u}_{1}(Y(t), z)$. As customary we do not distinguish between $u_{0}$ and $\tilde{u}_{0}, u_{1}$ and $\tilde{u}_{1}$. Then the controls $u_{0}$ and $u_{1}$ can simply be identified with functions $\tilde{u}_{0}(y)$ and $\tilde{u}_{1}(y, z)$, where $y \in \mathcal{S}$ and $z \in \mathbb{R}^{k}$.
When the control $u$ is Markovian, the corresponding process $Y^{(u)}(t)$ becomes a Markov process with the generator $A^{u}$ of $\phi \in C^{2}\left(\mathbb{R}^{k}\right)$ given by

$$
\begin{aligned}
A \phi(y)= & \sum_{i=1}^{k} \alpha_{i}\left(y, u_{0}(y)\right) \frac{\partial \phi}{\partial y_{i}}(y) \\
& +\frac{1}{2} \sum_{i, j=1}^{k}\left(\beta \beta^{T}\right)_{i, j}\left(y, u_{0}(y)\right) \frac{\partial^{2} \phi}{\partial y_{i} \partial y_{j}}(y) \\
& +\sum_{j=1}^{k} \int_{\mathbb{R}}\left\{\phi\left(y+\theta^{j}\left(y, u_{1}(y, z), z\right)\right)-\phi(y)\right. \\
& \left.-\nabla \phi(y) \theta^{j}\left(y, u_{1}(y, z), z\right)\right\} \nu(d z),
\end{aligned}
$$

where $\nabla \phi=\left(\partial \phi / \partial y_{1}, \ldots, \partial \phi / \partial y_{k}\right)$ is the gradient of $\phi$, and $\theta^{j}$ is the $j$ th column of the $k \times k$ matrix $\theta$.

Now we can state the main result of this section.

Theorem 3 (verification theorem for game (9) and (13)). Suppose there exist two functions $\phi_{i}: \overline{\mathcal{S}} \rightarrow \mathbb{R} ; i=1,2$ such that

(i) $\phi_{i} \in C^{1}\left(\mathcal{S}^{0}\right) \bigcap C(\overline{\mathcal{S}}), i=1,2$, where $\overline{\mathcal{S}}$ is the closure of $\mathcal{S}$ and $\mathcal{S}^{0}$ is the interior of $\mathcal{S}$;

(ii) $\phi_{i} \geq g_{i}$ on $\mathcal{S}, i=1,2$.

Define the following continuation regions:

$$
D_{i}=\left\{y \in \mathcal{S} ; \phi_{i}(y)>g_{i}(y)\right\}, \quad i=1,2 .
$$

Suppose $Y(t)=Y^{u}(t)$ spends 0 time on $\partial D_{i}$ a.s., $i=$ 1,2 , that is,

(iii) $E^{y}\left[\int_{0}^{\tau_{S}} \chi_{\partial D_{i}}(Y(t)) d t\right]=0$ for all $y \in \mathcal{S}, u \in \mathcal{U}, i=1,2$,

(iv) $\partial D_{i}$ is a Lipschitz surface, $i=1,2$,

(v) $\phi_{i} \in C^{2}\left(\mathcal{S} \backslash \partial D_{i}\right)$. The second-order derivatives of $\phi_{i}$ are locally bounded near $\partial D_{i}$, respectively, $i=1,2$,

(vi) $D_{1}=D_{2}:=D$,

(vii) there exists $\widehat{u} \in \mathscr{A}$ such that, for $i=1,2$,

$$
\begin{aligned}
& A^{\widehat{u}} \phi_{i}(y)+f_{i}(y, \widehat{u}(y)) \\
& \quad=\sup _{u \in \mathscr{A}}\left\{A^{u} \phi_{i}(y)+f_{i}(y, u(y))\right\} \begin{cases}=0, & y \in D, \\
\leq 0, & y \in \mathcal{S} \backslash D,\end{cases}
\end{aligned}
$$

(viii) $E^{y}\left[\left|\phi_{i}\left(Y_{\tau}\right)\right|+\int_{0}^{\tau}\left|A^{u} \phi_{i}\left(Y^{u}(t)\right)\right| d t\right]<\infty ; i=1,2$, for all $u \in \mathfrak{U}, \tau \in \Gamma$.

For $u \in \mathscr{A}$ define

$$
\tau_{D}=\tau_{D}^{u}=\inf \left\{t>0 ; Y^{u}(t) \notin D\right\}<\infty,
$$

and, in particular,

$$
\widehat{\tau}_{D}=\tau_{D}^{\widehat{u}}=\inf \left\{t>0 ; Y^{\widehat{u}}(t) \notin D\right\}<\infty .
$$




\section{Suppose that}

(ix) the family $\left\{\phi_{i}(Y(\tau)) ; \tau \in \Gamma, \tau \leq \tau_{D}\right\}$ is uniformly integrable, for all $u \in \mathscr{A}$ and $y \in \mathcal{S}, i=1,2$.

Then $\left(\widehat{\tau}_{D}, \widehat{u}\right) \in \Gamma \times \mathscr{A}$ is a Nash equilibrium for game (9), (13), and

$$
\begin{aligned}
& \phi_{1}(y)=\sup _{u \in \mathscr{A}} \mathcal{F}_{1}^{u, \hat{\tau}_{D}}(y)=\mathcal{J}_{1}^{\widehat{u}, \widehat{\tau}_{D}}(y), \\
& \phi_{2}(y)=\sup _{\tau \in \Gamma} \mathcal{F}_{2}^{\widehat{u}, \tau}(y)=\mathcal{J}_{2}^{\widehat{u}, \widehat{\tau}_{D}}(y) .
\end{aligned}
$$

Proof. From (i), (iv), and (v) we may assume by an approximation theorem (see Theorem 2.1 in [18]) that $\phi_{i} \in C^{2}(\mathcal{S})$, $i=1,2$.

For a given $u \in \mathscr{A}$, we define, with $Y(t)=Y^{u}(t)$,

$$
\tau_{D}=\tau_{D}^{u}=\inf \left\{t>0 ; Y^{u}(t) \notin D\right\} .
$$

In particular, let $\widehat{u}$ be as in (vii). Then,

$$
\widehat{\tau}_{D}=\tau_{D}^{\widehat{u}}=\inf \left\{t>0 ; Y^{\widehat{u}}(t) \notin D\right\} .
$$

We first prove that (21) holds. Let $\widehat{\tau}_{D} \in \Gamma$ be as in (24). For arbitrary $u \in \mathscr{A}$, by (vii) and the Dynkin's formula for jump diffusion (see Theorem 1.24 in [18]) we have

$$
\begin{aligned}
\phi_{1}(y) & =E^{y}\left[\int_{0}^{\widehat{\tau}_{D} \wedge m}-A^{u} \phi_{1}(Y(t)) d t+\phi_{1}\left(Y\left(\widehat{\tau}_{D} \wedge m\right)\right)\right] \\
& \geq E^{y}\left[\int_{0}^{\hat{\tau}_{D} \wedge m} f_{1}(Y(t), u(t)) d t+\phi_{1}\left(Y\left(\widehat{\tau}_{D} \wedge m\right)\right)\right],
\end{aligned}
$$

where $m=1,2, \ldots$. Therefore, by (11), (12), (i), (ii), (viii), and the Fatou lemma,

$$
\begin{aligned}
& \phi_{1}(y) \\
& \quad \geq \liminf _{m \rightarrow \infty} E^{y}\left[\int_{0}^{\widehat{\tau}_{D} \wedge m} f_{1}(Y(t), u(t)) d t+\phi_{1}\left(Y\left(\widehat{\tau}_{D} \wedge m\right)\right)\right] \\
& \geq E^{y}\left[\int_{0}^{\widehat{\tau}_{D}} f_{1}(Y(t), u(t)) d t+g_{1}\left(Y\left(\widehat{\tau}_{D}\right)\right)\right] \\
& =\mathscr{J}_{1}^{y}\left(u, \widehat{\tau}_{D}\right) .
\end{aligned}
$$

Since this holds for all $u \in \mathscr{A}$, we have

$$
\phi_{1}(y) \geq \sup _{u \in \mathscr{A}} \mathscr{F}_{1}^{y}\left(u, \widehat{\tau}_{D}\right) .
$$

In particular, applying the Dynkin's formula to $u=\widehat{u}$ we get an equality, that is,

$$
\begin{aligned}
\phi_{1}(y) & =E^{y}\left[\int_{0}^{\widehat{\tau}_{D} \wedge m}-A^{\widehat{u}} \phi_{1}(\widehat{Y}(t)) d t+\phi_{1}\left(\widehat{Y}\left(\widehat{\tau}_{D} \wedge m\right)\right)\right] \\
& =E^{y}\left[\int_{0}^{\widehat{\tau}_{D} \wedge m} f_{1}(\widehat{Y}(t), \widehat{u}(t)) d t+\phi_{1}\left(\widehat{Y}\left(\widehat{\tau}_{D} \wedge m\right)\right)\right],
\end{aligned}
$$

where $\widehat{Y}(t)=Y^{\widehat{u}}(t)$ and $m=1,2, \ldots$. Hence we deduce that

$$
\phi_{1}(y)=\mathscr{J}_{1}^{y}\left(\widehat{u}, \widehat{\tau}_{D}\right)
$$

Since we always have

$$
\mathscr{J}_{1}^{y}\left(\widehat{u}, \widehat{\tau}_{D}\right) \leq \sup _{u \in \mathscr{A}} \mathscr{F}_{1}^{y}\left(u, \widehat{\tau}_{D}\right)
$$

we conclude by combining (27), (29), and (30) that

$$
\phi_{1}(y)=\mathscr{J}_{1}^{y}\left(\widehat{u}, \widehat{\tau}_{D}\right)=\sup _{u \in \mathscr{A}} \mathscr{F}_{1}^{y}\left(u, \widehat{\tau}_{D}\right)
$$

which is (21).

Next we prove that (22) holds. Let $\widehat{u} \in \mathscr{A}$ be as in (vii). For $\tau \in \Gamma$, by the Dynkin's formula and (vii), we have

$$
\begin{aligned}
E^{y}\left[\phi_{2}\left(\widehat{Y}\left(\tau_{m}\right)\right)\right] & =\phi_{2}(y)+E^{y}\left[\int_{0}^{\tau_{m}} A^{\widehat{u}} \phi_{2}(\widehat{Y}(t)) d t\right] \\
& \leq \phi_{2}(y)-E^{y}\left[\int_{0}^{\tau_{m}} f_{2}(\widehat{Y}(t), \widehat{u}(t)) d t\right],
\end{aligned}
$$

where $\tau_{m}=\tau \wedge m ; m=1,2, \ldots$

Letting $m \rightarrow \infty$ gives, by (11), (12), (i), (ii), (viii), and the Fatou Lemma,

$$
\begin{aligned}
\phi_{2}(y) & \geq \liminf _{m \rightarrow \infty} E^{y}\left[\int_{0}^{\tau \wedge m} f_{2}(\widehat{Y}(t), \widehat{u}(t)) d t+\phi_{2}\left(\widehat{Y}\left(\tau_{m}\right)\right)\right] \\
& \geq E^{y}\left[\int_{0}^{\tau} f_{2}(\widehat{Y}(t), \widehat{u}(t)) d t+g_{2}(\widehat{Y}(\tau)) \chi_{\{\tau<\infty\}}\right] \\
& =\mathscr{J}_{2}^{y}(\widehat{u}, \tau) .
\end{aligned}
$$

The inequality (33) holds for all $\tau \in \Gamma$. Then we have

$$
\phi_{2}(y) \geq \sup _{\tau \in \Gamma} \mathscr{J}_{2}^{y}(\widehat{u}, \tau) .
$$

Similarly, applying the above argument to the pair $\left(\widehat{u}, \widehat{\tau}_{D}\right)$ we get an equality in (34), that is,

$$
\phi_{2}(y)=\mathscr{J}_{2}^{y}\left(\widehat{u}, \widehat{\tau}_{D}\right) .
$$

We always have

$$
\mathscr{J}_{2}^{y}\left(\widehat{u}, \widehat{\tau}_{D}\right) \leq \sup _{\tau \in \Gamma} \mathscr{F}_{2}^{y}(\widehat{u}, \tau) .
$$

Therefore, combining (34), (35), and (36) we get

$$
\phi_{2}(y)=\mathscr{J}_{2}^{y}\left(\widehat{u}, \widehat{\tau}_{D}\right)=\sup _{\tau \in \Gamma} \mathscr{J}_{2}^{y}(\widehat{u}, \tau),
$$

which is (22). The proof is completed. 


\section{An Example}

In this section we come back to Example 1 and use Theorem 3 to study the solutions of game (4) and (8). Here and in the following, all the processes are assumed to be one-dimension for simplicity.

To put game (4) and (8) into the framework of Section 2, we define the process $Y(t)=\left(Y_{0}(t), Y_{1}(t)\right) ; Y(0)=y=\left(s, y_{1}\right)$ by

$$
\begin{gathered}
d Y_{0}(t)=d t, \quad Y_{0}(0)=s \in \mathbb{R}, \\
d Y_{1}(t)=d X(t) \\
=Y_{1}(t)\{[(1-u(t)) r(t)+u(t) b(t)] d t \\
+u(t) \sigma(t) d B(t) \\
\left.+u(t-) \int_{\mathbb{R}} \gamma(t, z) \widetilde{N}(d t, d z)\right\}, \\
\quad Y_{1}(0)=x=y_{1} .
\end{gathered}
$$

Then the performance functionals to the controller (6) and the stopper (7) can be formulated as follows:

$$
\begin{gathered}
\mathscr{J}_{1}^{y}(u, \tau)=E^{s, y_{1}}\left[e^{-\delta(s+\tau)} U_{1}\left(C\left(Y_{1}(\tau)\right)\right)\right. \\
\left.-\int_{0}^{\tau} e^{-\delta(s+t)} h\left(Y_{0}(t), Y_{1}(t), u(t)\right) d t\right], \\
\mathscr{J}_{2}^{y}(u, \tau)=E^{s, y_{1}}\left[e^{-\delta(s+\tau)} U_{2}\left(Y_{1}(\tau)-C\left(Y_{1}(\tau)\right)\right)\right] .
\end{gathered}
$$

In this case the generator $A^{u}$ in (16) has the form

$$
\begin{aligned}
& A^{u} \phi(s, x) \\
& =\frac{\partial \phi}{\partial s}+[(1-u) r x+u b x] \frac{\partial \phi}{\partial x}+\frac{1}{2} x^{2} u^{2} \sigma^{2} \frac{\partial^{2} \phi}{\partial x^{2}} \\
& +\int_{\mathbb{R}_{0}}\left\{\phi(s, x+x u \gamma(z))-\phi(s, x)-x u \gamma(z) \frac{\partial \phi}{\partial x}\right\} \\
& \quad \times v(d z) .
\end{aligned}
$$

To obtain a possible Nash equilibrium $(\widehat{u}, \widehat{\tau}) \in \mathscr{A} \times \Gamma$ for game (4) and (8), according to Theorem 3 , it is necessary to find a subset $D$ of $\mathcal{S}=\mathbb{R}_{+}^{2}:=[0, \infty)^{2}$ and $\phi_{i}(s, x) ; i=1,2$, such that

(i) $\phi_{1}(s, x)=e^{-\delta s} U_{1}(C(x))$ and $\phi_{2}(s, x)=e^{-\delta s} U_{2}(x-$ $C(x))$, for all $(s, x) \in D$;

(ii) $\phi_{1}(s, x) \geq e^{-\delta s} U_{1}(C(x))$ and $\phi_{2}(s, x) \geq e^{-\delta s} U_{2}(x-$ $C(x))$, for all $(s, x) \in \mathcal{S}$;

(iii) $A^{u} \phi_{1}(s, x)-h(s, x, u) \leq 0$ and $A^{u} \phi_{2}(s, x) \leq 0$, for all $(s, x) \in \mathcal{S} \backslash D$ and $u$;

(iv) there exists $\widehat{u}$ such that $A^{\widehat{u}} \phi_{1}(s, x)-h(s, x, \widehat{u})=$ $A^{\widehat{u}} \phi_{2}(s, x)=0$, for all $(s, x) \in D$.
Imposing the first-order condition on $A^{u} \phi_{1}(s, x)-$ $h(s, x, u)$ and $A^{u} \phi_{2}(s, x)$, we get the following equations for the optimal control processes $\widehat{u}$ :

$$
\begin{aligned}
(b x-r x) & \frac{\partial \phi_{1}}{\partial x}(s, x)+x^{2} \sigma^{2} \widehat{u} \frac{\partial^{2} \phi_{1}}{\partial x^{2}}(s, x) \\
+ & \int_{\mathbb{R}} r \gamma(z)\left[\frac{\partial \phi_{1}}{\partial x}(s, x+r \widehat{u} \gamma(z))-\frac{\partial \phi_{1}}{\partial x}(s, x)\right] \\
& -\frac{\partial h}{\partial u}(s, x, \widehat{u})=0, \\
(b x-r x) & \frac{\partial \phi_{2}}{\partial x}(s, x)+x^{2} \sigma^{2} \widehat{u} \frac{\partial^{2} \phi_{2}}{\partial x^{2}}(s, x) \\
+ & \int_{\mathbb{R}} r \gamma(z)\left[\frac{\partial \phi_{2}}{\partial x}(s, x+r \widehat{u} \gamma(z))-\frac{\partial \phi_{2}}{\partial x}(s, x)\right]=0 .
\end{aligned}
$$

With $\widehat{u}$ as in (41), we put

$$
\begin{aligned}
& A^{\widehat{u}} \phi_{i}(s, x) \\
& =\frac{\partial \phi_{i}}{\partial s}+[(1-\widehat{u}) r x+\widehat{u} b x] \frac{\partial \phi_{i}}{\partial x}+\frac{1}{2} x^{2} \widehat{u}^{2} \sigma^{2} \frac{\partial^{2} \phi_{i}}{\partial x^{2}} \\
& \quad+\int_{\mathbb{R}_{0}}\left\{\phi_{i}(s, x+x \widehat{u} \gamma(z))-\phi_{i}(s, x)-x \widehat{u} \gamma(z) \frac{\partial \phi_{i}}{\partial x}\right\} \\
& \quad \times v(d z) .
\end{aligned}
$$

Thus, we may reduce game (4) and (8) to the problem of solving a family of nonlinear variational-integro inequalities. We summarize as follows.

Theorem 4. Suppose there exist $\widehat{u}$ satisfying (41) and two $C^{1}$ functions $\phi_{i} ; i=1,2$ such that

(1)

$$
\begin{aligned}
D & =\left\{(s, x): \phi_{2}(s, x)>e^{-\delta s} U_{2}(x-C(x))\right\} \\
& =\left\{(s, x): \phi_{1}(s, x)>e^{-\delta s} U_{1}(C(x))\right\} ;
\end{aligned}
$$

(2) $\phi_{i} \in C^{2}(D), i=1,2$;

(3) $\phi_{2}(s, x)=e^{-\delta s} U_{2}(x-C(x))$ and $\phi_{1}(s, x)=$ $e^{-\delta s} U_{1}(C(x))$ for all $(s, x) \in \mathcal{S} \backslash D$;

(4) $A^{u} \phi_{1}(s, x)-h(s, x, u) \leq 0$ and $A^{u} \phi_{2}(s, x) \leq 0$ for all $(s, x) \in \mathcal{S} \backslash D$ and for all $u \in \mathscr{A}$;

(5) $A^{\widehat{u}} \phi_{1}(s, x)-h(s, x, \widehat{u})=0$ for all $(s, x) \in D$, where $A^{\widehat{u}} \phi_{1}$ is given by (42);

(6) $A^{\widehat{u}} \phi_{2}(s, x)=0$ for all $(s, x) \in D$, where $A^{\widehat{u}} \phi_{2}$ is given by (42).

Then the pair $(\widehat{u}, \widehat{\tau})$ is a Nash equilibrium of the stochastic differential game (4) and (8), where

$$
\widehat{\tau}=\inf \left\{t>0 ; Y^{\widehat{u}}(t) \notin D\right\} .
$$


Moreover, the corresponding equilibrium performances are

$$
\begin{aligned}
& \phi_{1}(s, x)=\mathscr{J}_{1}^{(s, x)}(\widehat{u}, \widehat{\tau}), \\
& \phi_{2}(s, x)=\mathscr{J}_{2}^{(s, x)}(\widehat{u}, \widehat{\tau}) .
\end{aligned}
$$

In this paper we will not discuss general solutions of this family of nonlinear variational-integro inequalities. Instead we discuss a solution in special case when

$$
\gamma(t, z)=0, \quad h(s, x, u)=\frac{u^{2}}{2} .
$$

Let us try the functions $\phi_{i}, i=1,2$, of the form

$$
\phi_{i}(s, x)=e^{-\delta s} \psi_{i}(x),
$$

and a continuation region $D$ of the form

$$
D=\left\{(s, x) ; x<x_{0}\right\} \quad \text { for some } x_{0}>0 .
$$

Then we have

$$
A^{u} \phi_{i}(s, x)=e^{-\delta s} A^{u} \psi_{i}(x),
$$

where

$$
\begin{aligned}
A^{u} \psi_{i}(x)= & -\delta \psi_{i}(x)+[(1-u) r x+u b x] \psi_{i}^{\prime}(x) \\
& +\frac{1}{2} x^{2} u^{2} \sigma^{2} \psi_{i}^{\prime \prime}(x) .
\end{aligned}
$$

By conditions (1) and (3) in Theorem 4, we get

$$
\begin{gathered}
\psi_{1}(x)=U_{1}(C(x)), \quad x \geq x_{0}, \\
\psi_{1}(x)>U_{1}(C(x)), \quad 0<x<x_{0}, \\
\psi_{2}(x)=U_{2}(x-C(x)), \quad x \geq x_{0}, \\
\psi_{2}(x)>U_{2}(x-C(x)), \quad 0<x<x_{0} .
\end{gathered}
$$

From conditions (4), (5), and (6) of Theorem 4, we get the candidate $\widehat{u}$ for the optimal control as follows:

$$
\begin{aligned}
\widehat{u}=\underset{u \in \mathscr{A}}{\operatorname{Argmax}}\left\{A^{u} \psi_{1}(x)-\frac{u^{2}}{2}\right\} & \underset{u \in \mathscr{A}}{\operatorname{Argmax}}\left\{-\delta \psi_{1}(x)+[(1-u) r x+u b x] \psi_{1}^{\prime}(x)\right. \\
& \left.+\frac{1}{2} x^{2} u^{2} \sigma^{2} \psi_{1}^{\prime \prime}(x)-\frac{u^{2}}{2}\right\} \\
= & \frac{(b x-r x) \psi_{1}^{\prime}(x)}{1-x^{2} \sigma^{2} \psi_{1}^{\prime \prime}(x)}, \\
\widehat{u}= & \underset{u \in \mathscr{A}}{\operatorname{Argmax}}\left\{A^{u} \psi_{2}(x)\right\} \\
= & \underset{u \in \mathscr{A}}{\operatorname{Argmax}}\left\{-\delta \psi_{2}(x)+[(1-u) r x+u b x] \psi_{2}^{\prime}(x)\right. \\
= & \frac{-(b x-r x) \psi_{2}^{\prime}(x)}{x^{2} \sigma^{2} \psi_{2}^{\prime \prime}(x)} . \\
& \left.+\frac{1}{2} x^{2} u^{2} \sigma^{2} \psi_{2}^{\prime \prime}(x)\right\}
\end{aligned}
$$

Let $\psi_{i}(x)=\widetilde{\psi}_{i}(x)$ on $0<x<x_{0}, i=1,2$. By condition (5) in Theorem 4 , we have $A^{\widehat{u}} \widetilde{\psi}_{1}(x)-\widehat{u}^{2} / 2=0$ for $0<x<x_{0}$. Substituting (52) into $A^{u} \widetilde{\psi}_{1}(x)-u^{2} / 2=0$, we obtain

$$
\begin{aligned}
-\delta \widetilde{\psi}_{1}(x) & +\left[r x+\frac{(b x-r x)^{2} \widetilde{\psi}_{1}^{\prime}(x)}{1-x^{2} \sigma^{2} \widetilde{\psi}_{1}^{\prime \prime}(x)}\right] \widetilde{\psi}_{1}^{\prime}(x) \\
+ & \frac{\left[(b x-r x) \widetilde{\psi}_{1}^{\prime}(x)\right]^{2}}{2\left(1-x^{2} \sigma^{2} \widetilde{\psi}_{1}^{\prime \prime}(x)\right)^{2}}\left[x^{2} \sigma^{2} \widetilde{\psi}_{1}^{\prime \prime}(x)-1\right]=0 .
\end{aligned}
$$

Similarly, we obtain $A^{\widehat{u}} \widetilde{\psi}_{2}(x)=0$ for $0<x<x_{0}$ by condition (6) in Theorem 4 . And we substitute (53) in $A^{u} \widetilde{\psi}_{2}(x)=0$ to get

$$
\begin{gathered}
-\delta \widetilde{\psi}_{2}(x)+r x \widetilde{\psi}_{2}^{\prime}(x)+\frac{\left[(b x-r x) \widetilde{\psi}_{2}^{\prime}(x)\right]^{2}}{1-x^{2} \sigma^{2} \widetilde{\psi}_{2}^{\prime \prime}(x)} \\
+\frac{\left[x \sigma(b x-r x) \widetilde{\psi}_{2}^{\prime}(x)\right]^{2} \widetilde{\psi}_{2}^{\prime \prime}(x)}{2\left(1-x^{2} \sigma^{2} \widetilde{\psi}_{2}^{\prime \prime}(x)\right)^{2}}=0 .
\end{gathered}
$$

Therefore, we conclude that

$$
\begin{gathered}
\psi_{1}(x)= \begin{cases}U_{1}(C(x)), & x \geq x_{0}, \\
\widetilde{\psi}_{1}(x), & 0<x<x_{0},\end{cases} \\
\psi_{2}(x)= \begin{cases}U_{2}(x-C(x)), & x \geq x_{0}, \\
\widetilde{\psi}_{2}(x), & 0<x<x_{0},\end{cases}
\end{gathered}
$$

where $\widetilde{\psi}_{1}(x)$ and $\widetilde{\psi}_{2}(x)$ are the solutions of (56) and (57), respectively.

According to Theorem 4, we use the continuity and differentiability of $\psi_{i}$ at $x=x_{0}$ to determine $x_{0}, i=1,2$, that is,

$$
\begin{gathered}
\psi_{1}\left(x_{0}\right)=U_{1}\left(C\left(x_{0}\right)\right), \\
\psi_{1}^{\prime}\left(x_{0}\right)=U_{1}^{\prime}\left(C\left(x_{0}\right)\right) C^{\prime}\left(x_{0}\right), \\
\psi_{2}\left(x_{0}\right)=U_{2}\left(x_{0}-C\left(x_{0}\right)\right) \\
\psi_{2}^{\prime}\left(x_{0}\right)=U_{2}^{\prime}\left(x_{0}-C\left(x_{0}\right)\right)\left(1-C^{\prime}\left(x_{0}\right)\right) .
\end{gathered}
$$

At the end of this section, we summarize the above results in the following theorem.

Theorem 5. Let $\psi_{i}, i=1,2$ and let $x_{0}$ be the solutions of equations (56)-(58). Then the pair $(\widehat{u}, \widehat{\tau})$ given by

$$
\begin{gathered}
\widehat{u}=\frac{(b x-r x) \psi_{1}^{\prime}(x)}{1-x^{2} \sigma^{2} \psi_{1}^{\prime \prime}(x)}=\frac{-(b x-r x) \psi_{2}^{\prime}(x)}{x^{2} \sigma^{2} \psi_{2}^{\prime \prime}(x)}, \\
\widehat{\tau}=\inf \left\{t>0 ; x \geq x_{0}\right\}
\end{gathered}
$$

is a Nash equilibrium of game (4) and (8). The corresponding equilibrium performances are

$$
\phi_{i}(s, x)=e^{-\delta s} \psi_{i}(x), \quad i=1,2 .
$$




\section{Conclusion}

A verification theorem is obtained for the general stochastic differential game between a controller and a stopper. In the special case of quadratic cost, we use this theorem to characterize the Nash equilibrium. However, the question of the existence and uniqueness of Nash equilibrium for the game remains open. It will be considered in our subsequent work.

\section{Acknowledgment}

This work was supported by the National Natural Science Foundation of China under Grant 61273022 and 11171050.

\section{References}

[1] J. Bertoin, Lévy Processes, Cambridge University Press, Cambridge, UK, 1996.

[2] D. Applebaum, Lévy Processes and Stochastic Calculus, Cambridge University Press, Cambridge, UK, 2003.

[3] S. Mataramvura and B. Øksendal, "Risk minimizing portfolios and HJBI equations for stochastic differential games," Stochastics, vol. 80, no. 4, pp. 317-337, 2008.

[4] R. J. Elliott and T. K. Siu, "On risk minimizing portfolios under a Markovian regime-switching Black-Scholes economy," Annals of Operations Research, vol. 176, pp. 271-291, 2010.

[5] G. Wang and Z. Wu, "Mean-variance hedging and forwardbackward stochastic differential filtering equations," Abstract and Applied Analysis, vol. 2011, Article ID 310910, 20 pages, 2011.

[6] I. Karatzas and W. Sudderth, "Stochastic games of control and stopping for a linear diffusion," in Random Walk, Sequential Analysis and Related Topics: A Festschrift in Honor of Y.S. Chow, A. Hsiung, Z. L. Ying, and C. H. Zhang, Eds., pp. 100-117, World Scientific Publishers, 2006.

[7] S. Hamadène, "Mixed zero-sum stochastic differential game and American game options," SIAM Journal on Control and Optimization, vol. 45, no. 2, pp. 496-518, 2006.

[8] M. K. Ghosh, M. K. S. Rao, and D. Sheetal, "Differential games of mixed type with control and stopping times," Nonlinear Differential Equations and Applications, vol. 16, no. 2, pp. 143158, 2009.

[9] M. K. Ghosh and K. S. Mallikarjuna Rao, "Existence of value in stochastic differential games of mixed type," Stochastic Analysis and Applications, vol. 30, no. 5, pp. 895-905, 2012.

[10] I. Karatzas and Q. Li, "BSDE approach to non-zero-sum stochastic differential games of control and stopping," in Stochastic Processes, Finance and Control, pp. 105-153, World Scientific Publishers, 2012.

[11] J.-P. Lepeltier, "On a general zero-sum stochastic game with stopping strategy for one player and continuous strategy for the other," Probability and Mathematical Statistics, vol. 6, no. 1, pp. 43-50, 1985.

[12] I. Karatzas and W. D. Sudderth, "The controller-and-stopper game for a linear diffusion," The Annals of Probability, vol. 29, no. 3, pp. 1111-1127, 2001.

[13] A. Weerasinghe, "A controller and a stopper game with degenerate variance control," Electronic Communications in Probability, vol. 11, pp. 89-99, 2006.
[14] I. Karatzas and I.-M. Zamfirescu, "Martingale approach to stochastic differential games of control and stopping," The Annals of Probability, vol. 36, no. 4, pp. 1495-1527, 2008.

[15] E. Bayraktar, I. Karatzas, and S. Yao, "Optimal stopping for dynamic convex risk measures," Illinois Journal of Mathematics, vol. 54, no. 3, pp. 1025-1067, 2010.

[16] E. Bayraktar and V. R. Young, "Proving regularity of the minimal probability of ruin via a game of stopping and control," Finance and Stochastics, vol. 15, no. 4, pp. 785-818, 2011.

[17] F. Bagherya, S. Haademb, B. Øksendal, and I. Turpina, "Optimal stopping and stochastic control differential games for jump diffusions," Stochastics, vol. 85, no. 1, pp. 85-97, 2013.

[18] B. Øksendal and A. Sulem, Applied Stochastic Control of Jump Diffusions, Springer, Berlin, Germany, 2nd edition, 2007.

[19] Q. Lin, R. Loxton, K. L. Teo, and Y. H. Wu, "A new computational method for a class of free terminal time optimal control problems," Pacific Journal of Optimization, vol. 7, no. 1, pp. 63$81,2011$.

[20] Q. Lin, R. Loxton, K. L. Teo, and Y. H. Wu, "Optimal control computation for nonlinear systems with state-dependent stopping criteria," Automatica, vol. 48, no. 9, pp. 2116-2129, 2012.

[21] C. Jiang, Q. Lin, C. Yu, K. L. Teo, and G.-R. Duan, "An exact penalty method for free terminal time optimal control problem with continuous inequality constraints," Journal of Optimization Theory and Applications, vol. 154, no. 1, pp. 30-53, 2012. 


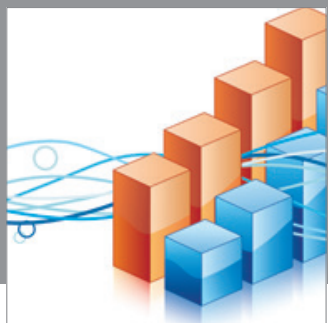

Advances in

Operations Research

mansans

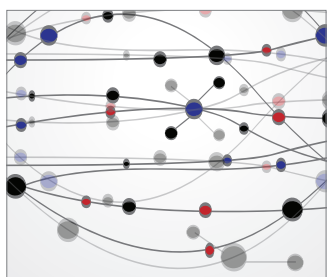

The Scientific World Journal
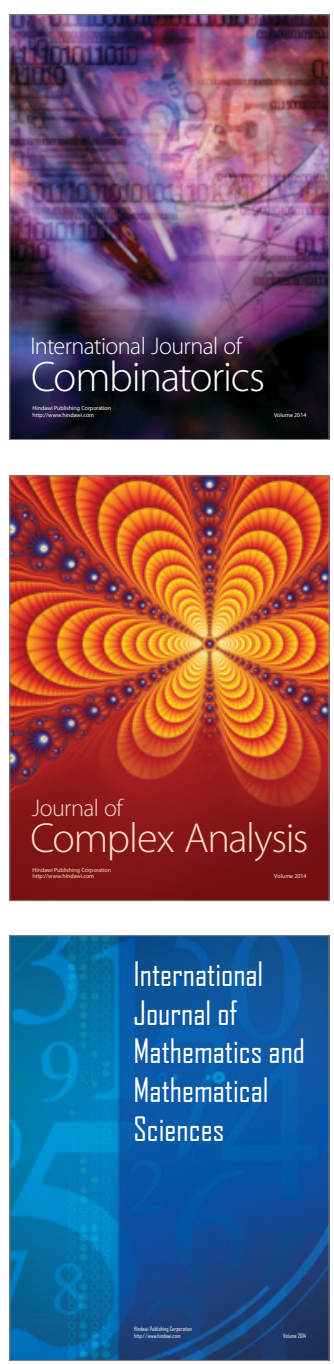
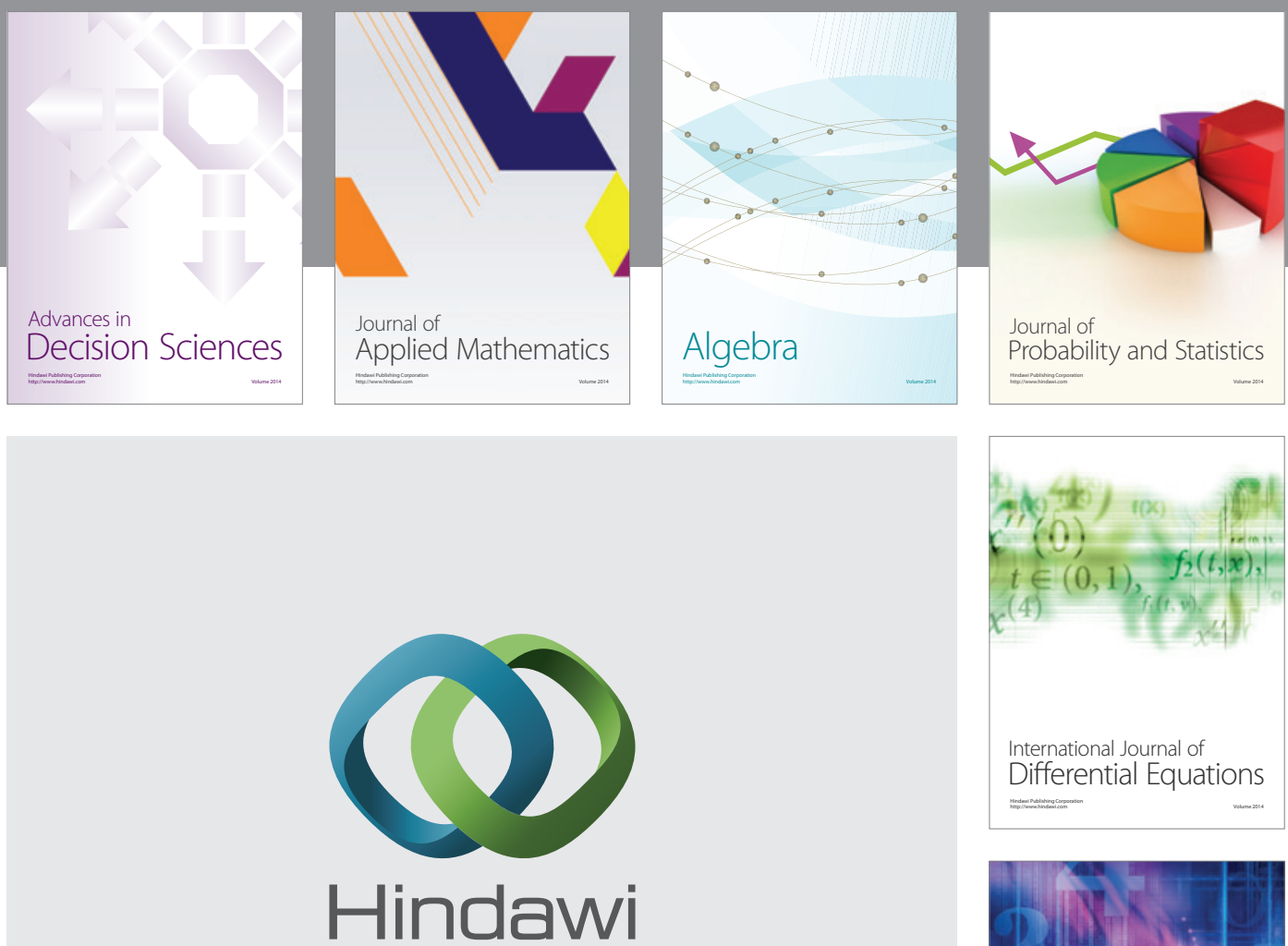

Submit your manuscripts at http://www.hindawi.com
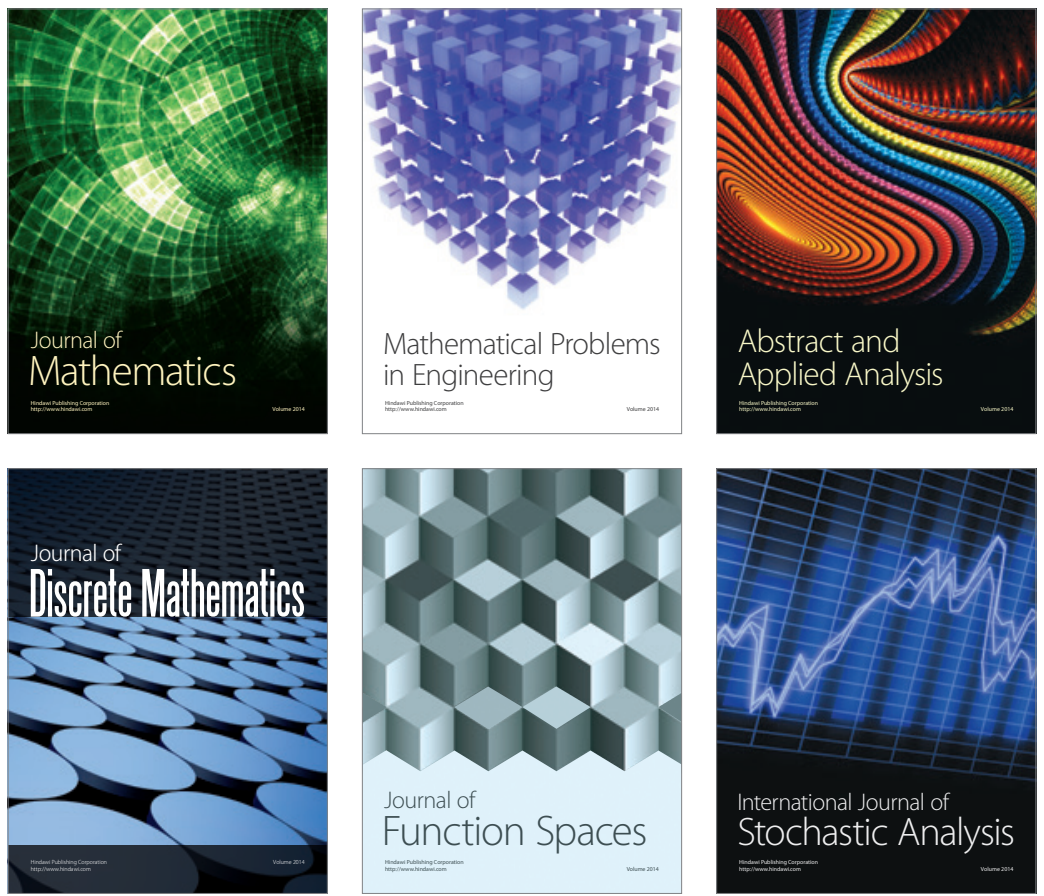

Journal of

Function Spaces

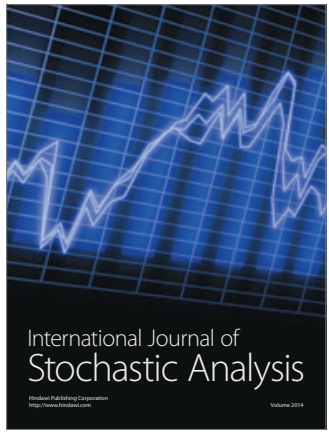

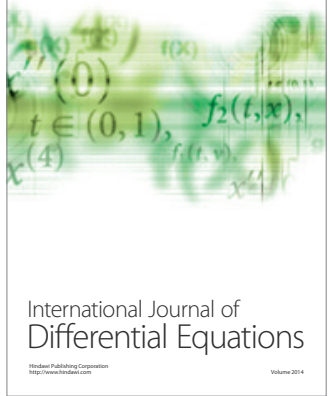
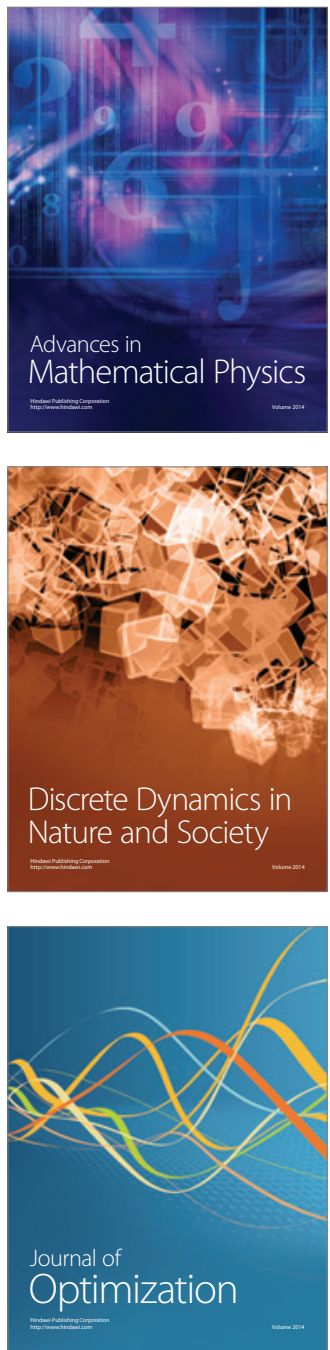